\title{
ON SOLUTIONS OF SEMILINEAR ELLIPTIC EQUATION WITH LINEAR GROWTH NONLINEARITY IN $\mathbb{R}^{N}$
}

\author{
Rong Cheng - Jianhua Hu
}

\begin{abstract}
We study nontrivial solutions for a class of semilinear elliptic equation which could be resonant at infinity. We establish the existence of solutions for the equation by considering the modified non-resonant problem associated with the original equation through Morse theory. Moreover, only linear growth assumption is imposed on the nonlinearity and condition on the potential is weaker than the coercive assumption.
\end{abstract}

\section{Introduction}

In the present paper, we are concerned with solutions of the following semilinear elliptic equation

$$
-\Delta u+\alpha(x) u=f(x, u), \quad x \in \mathbb{R}^{N}
$$

where the potential $\alpha(x) \in C\left(\mathbb{R}^{N}, \mathbb{R}\right)$ satisfies $\alpha(x)>\inf _{\mathbb{R}^{N}} \alpha(x)>0$. As shown in [3], [17], such a problem is motivated by the study of existence of standing wave solution for the nonlinear Schrödinger equation which arises in mathematical models from several physical phenomena, especially in nonlinear optics.

If we consider problem (1.1) in a bounded domain $\Omega \subset \mathbb{R}^{N}$, the compact condition will be assured by Sobolev Embedding Theorem for bounded domains. Such case was studied by many authors in many literatures and references cited

2010 Mathematics Subject Classification. 35J50, 37J45.

Key words and phrases. Critical points, semilinear elliptic equation, Morse theory.

Supported by Natural Science Foundation of the Jiangsu Higher Education Institutions (12KJB110015). 
there [16], [23], [12], [11], [18]. When the problem (1.1) is dealt with on entire $\mathbb{R}^{N}$, the main difficulty occurs for lack of compact condition, since $\mathbb{R}^{N}$ is unbounded. To overcome this difficulty, lots of assumptions were imposed on the potential $\alpha(x)$. Also the spectrum of the Schrödinger operator $\mathcal{L}=-\Delta+\alpha$ was studied in $[1],[2],[7],[6],[14],[10],[21]$.

In [19], the author considered a similar equation to (1.1) and make a different condition assumption on the potential which insures the compactness. Some different assumptions on the potential are employed in [5], [8]. In [17], [5], $\alpha(x)$ is assumed coercive, i.e. $\alpha(x) \rightarrow \infty$ as $|x| \rightarrow \infty$. This condition was generalized in [3]. That is

$$
\text { For every } M>0, \operatorname{mes}\left(\left\{x \in \mathbb{R}^{N} \mid \alpha(x) \leq M\right\}\right)<\infty,
$$

where $\operatorname{mes}(\cdot)$ stands for the Lebesgue measure in $\mathbb{R}^{N}$. The above assumption was used in [8] as well. The nonlinearity $f$ in [8] is asymptotically linear and satisfies some resonant conditions. In this paper, assumption on the potential $\alpha(x)$ is little different from [8] and we only need that $f$ has linear growth. Precisely, we assume that

$\left(\mathrm{H}_{1}\right) \alpha(x) \in C\left(\mathbb{R}^{N}, \mathbb{R}\right), \inf _{\mathbb{R}^{N}} \alpha(x)>0$ and there exists $r>0$ such that, for any $\gamma>0$

$$
\lim _{|y| \rightarrow \infty} \operatorname{mes}\left(A_{\gamma}(y)\right)=0
$$

where $A_{\gamma}(y)=\left\{x \in \mathbb{R}^{N} \mid \alpha(x)<\gamma\right\} \cap B_{r}(y)$, and $B_{r}(y)$ is a ball centered at $y$ with radius $r$.

$\left(\mathrm{H}_{2}\right) f(x, u) \in C^{1}\left(\mathbb{R}^{N} \times \mathbb{R}, \mathbb{R}\right)$ and there exists a constant $C>0$ such that

$$
|f(x, u)| \leq C|u| \quad \text { for } x \in \mathbb{R}^{N} \text { and } u \in \mathbb{R} \text {. }
$$

REMARK 1.1. Note that condition $\lim _{|y| \rightarrow \infty} \operatorname{mes}\left(A_{\gamma}(y)\right)=0$ in $\left(H_{1}\right)$ is weaker than (1.2). (1.2) implies for any $\gamma>0,\left\{x \in \mathbb{R}^{N} \mid \alpha(x)<\gamma\right\}$ is a bounded set. Therefore for $|y|$ large enough, $\left\{x \in \mathbb{R}^{N} \mid \alpha(x)<\gamma\right\} \cap B_{r}(y)=\emptyset$.

REMARK 1.2. The condition $\left(\mathrm{H}_{1}\right)$ is easy to verify. Let us take an instance for $N=1$. Take $\alpha(x)=e^{|x|}$. Then $\alpha(x) \in C\left(\mathbb{R}^{1}, \mathbb{R}\right), \inf _{\mathbb{R}^{1}} \alpha(x)=1>0$. Moreover, for $r=1$ and any $\gamma>0, \lim _{|y| \rightarrow \infty} \operatorname{mes}\left(A_{\gamma}(y)\right)=0$, since for $|y|>\ln \gamma+1$, $\left\{x \in \mathbb{R}^{1} \mid \alpha(x)<\gamma\right\} \cap B_{r}(y)=\emptyset$.

We would not impose any resonant or non-resonant condition on the nonlinearity at infinity. The main idea is to study the interaction between the nonlinearity at infinity and linear spectrum directly. This method was employed to study the problem (1.1) in a bounded domain $\Omega \subset \mathbb{R}^{N}$ and the potential vanishes in [13]. Therefore the results in this paper generalizes some results of $[13]$. 
We work in a subspace of $H^{1}\left(\mathbb{R}^{N}\right)$ defined by

$$
E=\left\{u \in H^{1}\left(\mathbb{R}^{N}\right) \mid \int_{\mathbb{R}^{N}}\left(|\nabla u|^{2}+\alpha(x) u^{2}\right) d x<\infty\right\} .
$$

In order to state our main result, we first decompose $E$ as follows. For some function $\beta(x) \in C\left(\mathbb{R}^{N}, \mathbb{R}\right)$, we set

$$
E=E^{-}(\beta) \oplus E^{0}(\beta) \oplus E^{+}(\beta),
$$

where the operator $-\Delta-\beta$ is negatively definite on $E^{-}(\beta)$, positively definite on $E^{+}(\beta)$ and null on $E^{0}(\beta)$. Denote by $i(\beta)$ and $n(\beta)$ the dimension of $E^{-}(\beta)$ and $E^{0}(\beta)$, respectively.

Now the main result in this paper can be read as follows.

Theorem 1.3. Assume that $\left(\mathrm{H}_{1}\right),\left(\mathrm{H}_{2}\right)$ hold and $n\left(f_{u}^{\prime}(x, 0)\right)=0$. Then (1.1) possesses at least one nontrivial solution, provided one of the following conditions holds:

$\left(\mathrm{H}_{3}\right)$ There exists a constant $\tau>0$, and some function $\beta_{\infty}(x) \in C\left(\mathbb{R}^{N}, \mathbb{R}\right)$ such that $f_{u}^{\prime}(x, u) \leq \beta_{\infty}(x)+\alpha(x)$ for all $x \in \mathbb{R}^{N}$ and $u$ with $|u| \geq \tau$ and $i\left(\beta_{\infty}\right) \leq i\left(f_{u}^{\prime}(x, 0)\right)-2$.

$\left(\mathrm{H}_{4}\right)$ There exists a constant $\tau>0$, and some function $\beta_{\infty}(x) \in C\left(\mathbb{R}^{N}, \mathbb{R}\right)$ such that $f_{u}^{\prime}(x, u) \geq \beta_{\infty}(x)+\alpha(x)$ for all $x \in \mathbb{R}^{N}$ and $u$ with $|u| \geq \tau$ and $i\left(\beta_{\infty}\right) \geq i\left(f_{u}^{\prime}(x, 0)\right)+2$.

REMARK 1.4. Resonant condition or non-resonant condition at infinity for the nonlinearity $f$ are often supposed in the references. From Theorem 1.3, it is easy to see that we do not impose any resonant or non-resonant condition on the nonlinearity $f$ at infinity.

\section{Proof of the main result}

It is well known that the space $E$ equipped with the following inner product

$$
\langle u, v\rangle=\int_{\mathbb{R}^{N}}(\nabla u \nabla v+\alpha(x) u v) d x
$$

is a Hilbert space. Denote by $\|\cdot\|$ the associated norm, i.e.

$$
\|u\|^{2}=\int_{\mathbb{R}^{N}}\left(|\nabla u|^{2}+\alpha(x) u^{2}\right) d x .
$$

By $\left(\mathrm{H}_{2}\right)$ and [22], the variational functional

$$
I(u)=\frac{1}{2} \int_{\mathbb{R}^{N}}\left(|\nabla u|^{2}+\alpha(x) u^{2}\right) d x-\int_{\mathbb{R}^{N}} F(x, u) d x
$$

is well defined and of the class $C^{2}$, where $F(x, u)=\int_{0}^{u} f(x, s) d s$. Furthermore, the critical points of $I$ are precisely the weak solutions of equation (1.1).

By the condition (1.2) and the Sobolev Embedding Theorem, the immersion $E \hookrightarrow L^{p}\left(\mathbb{R}^{N}\right)$ is compact for $2 \leq p<2^{*}=2 N /(N-2)$, which is proved in [3]. 
Modify the proof in [3], we can show the embedding $E \hookrightarrow L^{p}\left(\mathbb{R}^{N}\right)$ is also compact for $2 \leq p<2^{*}$ under the assumption $\left(\mathrm{H}_{1}\right)$.

Lemma 2.1. Under the condition $\left(\mathrm{H}_{1}\right)$, the embedding $E \hookrightarrow L^{p}\left(\mathbb{R}^{N}\right)$ is compact for $2 \leq p<2^{*}$.

Proof. We first consider the case where $p=2$. Let $\left\{u_{n}\right\} \subset E$ such that $\left\|u_{n}\right\| \leq C_{0}$. Then up to a subsequence, we have $u_{n}$ converges weakly to $u$ in $E$ as $n \rightarrow \infty$. We want to show that

$$
u_{n} \rightarrow u \quad \text { in } L^{2}\left(\mathbb{R}^{N}\right), \quad \text { as } n \rightarrow \infty .
$$

Define $v_{n}=u_{n}-u$. Then $v_{n} \rightarrow 0$ in $E$. We only need to prove $v_{n} \rightarrow 0$ in $L^{2}\left(\mathbb{R}^{N}\right)$. By the Sobolev Embedding Theorem for bounded domains, one has $v_{n} \rightarrow 0$ in $L^{2}\left(B_{R}\right)$. The remain is to estimate $\int_{B_{R}^{c}} v_{n}^{2} d x$. We first choose $\left\{y_{j}\right\} \subset \mathbb{R}^{N}$ such that $\mathbb{R}^{N} \subset \bigcup_{j=1}^{\infty} B_{r}\left(y_{j}\right)$ and each $x \in \mathbb{R}^{N}$ is covered by at most $2^{N}$ such balls.

Therefore

$$
\begin{aligned}
\int_{B_{R}^{c}} v_{n}^{2} d x & \leq \sum_{\left|y_{j}\right|>R-r}^{\infty} \int_{B_{r}\left(y_{j}\right)} v_{n}^{2} d x \\
& =\sum_{\left|y_{j}\right|>R-r}^{\infty} \int_{B_{r}\left(y_{j}\right) \cap\left\{x \in \mathbb{R}^{N} \mid \alpha(x)>\gamma\right\}} v_{n}^{2} d x+\int_{A_{\gamma}\left(y_{j}\right)} v_{n}^{2} d x .
\end{aligned}
$$

Then

$$
\int_{B_{r}\left(y_{j}\right) \cap\left\{x \in \mathbb{R}^{N} \mid \alpha(x)>\gamma\right\}} v_{n}^{2} d x \leq \frac{1}{\gamma} \int_{B_{r}\left(y_{j}\right)} \alpha(x) v_{n}^{2} d x .
$$

and by Hölder inequality, one has

$$
\begin{aligned}
\int_{A_{\gamma}\left(y_{j}\right)} v_{n}^{2} d x & \leq\left(\int_{A_{\gamma}\left(y_{j}\right)} v_{n}^{2 N /(N-2)} d x\right)^{(N-2) / N}\left(\int_{A_{\gamma}\left(y_{j}\right)} 1 d x\right)^{2 / N} \\
& \leq\left\|v_{n}\right\|_{L^{2^{*}\left(B_{r}\left(y_{j}\right)\right)}}^{2}\left[\operatorname{mes}\left(A_{\gamma}\left(y_{j}\right)\right)\right]^{2 / N} \\
& \leq\left\|v_{n}\right\|_{H^{1}\left(B_{r}\left(y_{j}\right)\right)}^{2} \sup _{\left|y_{j}\right| \geq R-r}\left[\operatorname{mes}\left(A_{\gamma}\left(y_{j}\right)\right)\right]^{2 / N} .
\end{aligned}
$$

We have then

$$
\begin{aligned}
\int_{B_{R}^{c}} v_{n}^{2} d x \leq & \sum_{\left|y_{j}\right| \geq R-r}^{\infty}\left[\frac{1}{\gamma} \int_{B_{r}\left(y_{j}\right)} \alpha(x) v_{n}^{2} d x\right. \\
& \left.+C_{1} \sup _{\left|y_{j}\right| \geq R-r}\left[\operatorname{mes}\left(A_{\gamma}\left(y_{j}\right)\right)\right]^{2 / N} \int_{B_{r}\left(y_{j}\right)}\left(\left|\nabla v_{n}\right|^{2}+\alpha(x) v_{n}^{2}\right) d x\right] \\
\leq & \frac{2^{N}}{\gamma} \int_{B_{R-2 r}^{c}} \alpha(x) v_{n}^{2} d x
\end{aligned}
$$




$$
\begin{aligned}
& +2^{N} C_{1} \sup _{\left|y_{j}\right| \geq R-r}\left[\operatorname{mes}\left(A_{\gamma}\left(y_{j}\right)\right)\right]^{2 / N} \int_{B_{R-2 r}^{c}}\left(\left|\nabla v_{n}\right|^{2}+\alpha(x) v_{n}^{2}\right) d x \\
\leq & \frac{2^{N}}{\gamma} C_{0}^{2}+C_{1} \sup _{\left|y_{j}\right| \geq R-r}\left[\operatorname{mes}\left(A_{\gamma}\left(y_{j}\right)\right)\right]^{2 / N} C_{0}^{2} .
\end{aligned}
$$

For any $0<\varepsilon \ll 1$, we chose $\gamma$ such that $2^{N} C_{0}^{2} / \gamma<\varepsilon$. For such a fixed $\gamma>0$, there exists $R>0$ such that

$$
C_{1} \sup _{|y| \geq R-r}\left[\operatorname{mes}\left(A_{\gamma}\left(y_{j}\right)\right)\right]^{2 / N} C_{0}^{2}<\varepsilon .
$$

Since

$$
\sup _{\left|y_{j}\right| \geq R-r}\left[\operatorname{mes}\left(A_{\gamma}\left(y_{j}\right)\right)\right]^{2 / N} \rightarrow 0 \quad \text { as } R \rightarrow \infty .
$$

Thus for such $R>0$

$$
\int_{B_{R}^{c}} v_{n}^{2} d x \rightarrow 0 \text { as } n \rightarrow \infty .
$$

For $2<p<2^{*}$, by Hölder inequality and Sobolev inequality, one has

$$
\left\|v_{n}\right\|_{L^{p}} \leq\left.\left.\left\|v_{n}\right\|\right|_{L^{p}} ^{t}\left\|v_{n}\right\|\right|_{L^{2^{*}}} ^{1-t} \leq\left. C_{1}^{1-t}\left\|v_{n}\right\|_{L^{2}}^{t}\left\|v_{n}\right\|\right|^{1-t} \leq\left(C_{0} C_{1}\right)^{1-t}\left\|v_{n}\right\|_{L^{2}}^{t} \rightarrow 0
$$

as $n \rightarrow \infty$, where $t \in(0,1)$ satisfies $1 / p=t / 2+(1-t) / 2^{*}$. Then the proof is complete.

We recall that a sequence $\left\{u_{n}\right\} \subset E$ is said to be a (PS) sequence if $I\left(u_{n}\right)$ being bounded and $I^{\prime}\left(u_{n}\right) \rightarrow 0$. And $I$ satisfies (PS) condition if every (PS) sequence has a convergent subsequence. By Lemma 2.1, if we want to show $I$ satisfies (PS) condition, we only need to verify that $\left\{u_{n}\right\} \subset E$ is bounded. For a non-resonant elliptic problem, this can be done by a standard argument.

Now, let us recall some definitions on Morse theory [4], [15] which will be used later. Let $u$ be a critical point of $I$. The Morse index of $u$ is defined as the supremum of the subspace of $E$ on which $I^{\prime \prime}(u)$ is negative definite and is denoted by $\mu(u)$. The nullity of $u$ is defined as dimension of $\operatorname{ker} I^{\prime \prime}(u)$ and is denoted by $\nu(u)$. For a constant $c \in \mathbb{R}$, the level set of $I$ is defined as

$$
I_{c}=\{u \in E \mid I(u) \leq c\} .
$$

Let $H_{q}(A, B)$ be the $q$-th singular homology group of the pair $(A, B)$. Then the Betti number $B_{q}$ of the pair $(A, B)$ is defined by $B_{q}=\operatorname{rank}\left(H_{q}(A, B)\right)$. For $a, b \in \mathbb{R}$ with $a<b$, the Morse type number $M_{q}$ of the pair $\left(I_{b}, I_{a}\right)$ is defined by

$$
M_{q}=\sum_{k=1}^{j} \operatorname{dim} C_{q}\left(I, u_{k}\right),
$$

where $C_{q}\left(I, u_{k}\right)$ is the $q$-th critical group and $u_{1}, \ldots, u_{j}$ are critical points contained in $I^{-1}([a, b])$. 
Lemma 2.2. Assume that $\left(\mathrm{H}_{1}\right)$ and $\left(\mathrm{H}_{2}\right)$ hold. And there exists a constant $\tau>0$ and some function $\beta(x) \in C\left(\mathbb{R}^{N}, \mathbb{R}\right)$ such that $f_{u}^{\prime}(x, u) \leq \beta(x)+\alpha(x)$ for all $x \in \mathbb{R}^{N}$ and $u$ with $|u| \geq \tau$. Then there exists a constant $\kappa=\kappa(\tau, \beta, C)$ such that for each solution $u$ of $(1.1),\|u\|_{L^{\infty}} \leq \kappa$, provided $\mu(u)+\nu(u) \geq$ $i(\beta)+n(\beta)+1$.

Proof. If the result is not true, then for any $n$, there exists a function $f_{n}$ and a $u_{n}$ satisfying

$$
-\Delta u_{n}+\alpha(x) u_{n}=f_{n}\left(x, u_{n}\right), \quad x \in \mathbb{R}^{N}
$$

such that $\mu\left(u_{n}\right)+\nu\left(u_{n}\right) \geq i(\beta)+n(\beta)+1$ and $\|u\|_{L^{\infty}} \geq n$. The corresponding functional is defined as

$$
I_{n}\left(u_{n}\right)=\frac{1}{2} \int_{\mathbb{R}^{N}}\left(\left|\nabla u_{n}\right|^{2}+\alpha(x) u_{n}^{2}\right) d x-\int_{\mathbb{R}^{N}} F_{n}\left(x, u_{n}\right) d x,
$$

where $F_{n}(x, u)=\int_{0}^{u} f_{n}(x, s) d s$. Then by $\left(\mathrm{H}_{1}\right)$ and elliptic estimate, we have

$$
\int_{\Omega \subset \mathbb{R}^{N}}\left|\nabla u_{n}\right|^{2} d x \rightarrow \infty \quad \text { as } n \rightarrow \infty \text {. }
$$

Thus

$$
\left\|u_{n}\right\| \geq \int_{\mathbb{R}^{N}}\left|\nabla u_{n}\right|^{2} d x \geq \int_{\Omega \subset \mathbb{R}^{N}}\left|\nabla u_{n}\right|^{2} d x \rightarrow \infty .
$$

Write $w_{n}=u_{n} /\left\|u_{n}\right\|$. Then $w_{n}$ is bounded with $\left\|w_{n}\right\|=1$. By passing to a subsequence, we can assume that for some $w \in E$

$$
\begin{array}{cl}
w_{n} \rightarrow w & \text { in } E, \\
w_{n} \rightarrow w & \text { in } L^{2}\left(\mathbb{R}^{N}\right), \\
w_{n}(x) \rightarrow w(x) & \text { a.e. } x \in \mathbb{R}^{N} .
\end{array}
$$

Then (2.1) multiplied by $u_{n}$ and integrated in $\mathbb{R}^{N}$, one has by $\left(\mathrm{H}_{2}\right)$

$$
\left\|u_{n}\right\|^{2}=\frac{1}{2} \int_{\mathbb{R}^{N}}\left(\left|\nabla u_{n}\right|^{2}+\alpha(x) u_{n}^{2}\right) d x=\int_{\mathbb{R}^{N}} f_{n}\left(x, u_{n}\right) u_{n} d x \leq C\left\|u_{n}\right\|_{L^{2}}^{2}
$$

which yields that $\|w\|_{L^{2}} \geq 1 / \sqrt{C}$ for each $n$, since

$$
\left\|w_{n}\right\|_{L^{2}}^{2}=\frac{1}{\left\|u_{n}\right\|^{2}} \int_{\mathbb{R}^{N}}\left|u_{n}\right|^{2} d x=\frac{1}{\left\|u_{n}\right\|^{2}}\left\|u_{n}\right\|_{L^{2}}^{2} \geq \frac{\left\|u_{n}\right\|_{L^{2}}^{2}}{C\left\|u_{n}\right\|_{L^{2}}^{2}}=\frac{1}{C} .
$$

By $\left(\mathrm{H}_{2}\right)$, for $u_{n} \neq 0, f\left(x, u_{n}\right) / u_{n}(x) \leq C$. Thus

$$
g_{n}(x)= \begin{cases}\frac{f_{n}\left(x, u_{n}\right)}{u_{n}} & \text { as } u_{n} \neq 0, \\ 0 & \text { as } u_{n}=0,\end{cases}
$$

is bounded in $L^{\infty}\left(\mathbb{R}^{N}\right)$. So by passing to a subsequence, we may assume

$$
g_{n}(x) \rightarrow g(x) \quad \text { in } L^{\infty}\left(\mathbb{R}^{N}\right)
$$


in weak* topology. Since $u_{n}$ satisfies $(2.1)$, we have for each $\varphi \in C_{0}^{\infty}\left(\mathbb{R}^{N}\right)$

$$
\int_{\mathbb{R}^{N}}\left(\nabla u_{n} \nabla \varphi+\alpha(x) u_{n} \varphi\right) d x-\int_{\mathbb{R}^{N}} f_{n}\left(x, u_{n}\right) \varphi d x=0 .
$$

Then, divided by $\left\|u_{n}\right\| \neq 0$, we have

$$
\int_{\mathbb{R}^{N}}\left(\nabla w_{n} \nabla \varphi+\alpha(x) w_{n} \varphi\right) d x-\int_{\mathbb{R}^{N}} \frac{f_{n}\left(x, u_{n}\right)}{u_{n}} \frac{u_{n}}{\left\|u_{n}\right\|} \varphi d x=0 .
$$

That is

$$
\int_{\mathbb{R}^{N}}\left(\nabla w_{n} \nabla \varphi+\alpha(x) w_{n} \varphi\right) d x-\int_{\mathbb{R}^{N}} g_{n}(x) w_{n} \varphi d x=0 .
$$

Let $n \rightarrow \infty$, one has

$$
\int_{\mathbb{R}^{N}}(\nabla w \nabla \varphi+\alpha(x) w \varphi) d x-\int_{\mathbb{R}^{N}} g(x) w \varphi d x=0 .
$$

It concludes that $w$ solves the following linear equation

$$
\left\{\begin{array}{l}
-\Delta w+\alpha(x) w=g(x) w, \\
x \in \mathbb{R}^{N} .
\end{array}\right.
$$

By the unique continuation property in [9], we have $w(x) \neq 0$ almost everywhere in $\mathbb{R}^{N}$, since $w \neq 0$. This means $u_{n}(x) \rightarrow \infty$ almost everywhere in $\mathbb{R}^{N}$.

Next we prove that there exists $n_{0}$, for any $z \in E^{+}(\beta) \backslash\{0\}$

$$
\left\langle I_{n}^{\prime \prime}\left(u_{n}\right) z, z\right\rangle>0 \quad \text { as } n \geq n_{0}
$$

which means $\mu\left(u_{n}\right)+\nu\left(u_{n}\right) \leq i(\beta)+n(\beta)$. That is a contradiction. Now, if (2.2) is not true, then there exists $n_{j} \rightarrow \infty$ and $z_{j} \in E^{+}(\beta)$ with $\left\|z_{j}\right\|=1$ such that $\left\langle I_{n_{j}}^{\prime \prime}\left(u_{n_{j}}\right) z_{j}, z_{j}\right\rangle \leq 0$, i.e.

$$
\int_{\mathbb{R}^{N}}\left(\left|\nabla z_{j}\right|^{2}+\alpha(x) z_{j}^{2}\right) d x-\int_{\mathbb{R}^{N}} f_{n_{j}, u}^{\prime}\left(x, u_{n_{j}}(x)\right) z_{j}^{2}(x) d x \leq 0 .
$$

That is

$$
\int_{\mathbb{R}^{N}} f_{n_{j}, u}^{\prime}\left(x, u_{n_{j}}(x)\right) z_{j}^{2}(x) d x \geq \int_{\mathbb{R}^{N}}\left(\left|\nabla z_{j}\right|^{2}+\alpha(x) z_{j}^{2}\right) d x=\left\|z_{j}\right\|^{2}=1 .
$$

Note that $\left\{z_{j}\right\}$ is bounded in $E$, by Lemma 2.1, we can assume that $z_{j} \rightarrow z$ in $L^{2}\left(\mathbb{R}^{N}\right)$. Then by Hölder inequality and Fatou's Lemma

$$
\begin{aligned}
\limsup _{j \rightarrow \infty} \int_{\mathbb{R}^{N}} f_{n_{j}, u}^{\prime}\left(x, u_{n_{j}}(x)\right) z_{j}^{2}(x) d x & =\limsup _{j \rightarrow \infty}\left[\int_{\mathbb{R}^{N}} f_{n_{j}, u}^{\prime}\left(x, u_{n_{j}}(x)\right)\left(z_{j}^{2}(x)-z^{2}(x)\right) d x\right. \\
& \left.+\int_{\mathbb{R}^{N}} f_{n_{j}, u}^{\prime}\left(x, u_{n_{j}}(x)\right) z^{2}(x) d x\right] \\
\leq & +\limsup _{j \rightarrow \infty} \int_{\mathbb{R}^{N}} f_{n_{j}, u}^{\prime}\left(x, u_{n_{j}}(x)\right) z^{2}(x) d x
\end{aligned}
$$




$$
\begin{aligned}
& \leq \int_{\mathbb{R}^{N}} \limsup _{j \rightarrow \infty} f_{n_{j}, u}^{\prime}\left(x, u_{n_{j}}(x)\right) z^{2}(x) d x \\
& \leq \int_{\mathbb{R}^{N}}(\alpha(x)+\beta(x)) z^{2}(x) d x<1
\end{aligned}
$$

since for $z \in E^{+}(\beta)$,

$$
\int_{\mathbb{R}^{N}}((-\Delta+\alpha(x)-\alpha(x)-\beta(x)) z, z)=\int_{\mathbb{R}^{N}}((-\Delta-\beta(x)) z, z)>0,
$$

that is

$$
\int_{\mathbb{R}^{N}}(\alpha(x)+\beta(x)) z^{2}(x) d x<\int_{\mathbb{R}^{N}}((-\Delta+\alpha(x)) z, z) d x=\|z\|^{2} .
$$

This contributes a contradiction and (2.2) is true.

Lemma 2.3. Assume $\left(\mathrm{H}_{1}\right),\left(\mathrm{H}_{2}\right)$ and there exists a constant $\tau>0$ and some function $\beta(x) \in C\left(\mathbb{R}^{N}, \mathbb{R}\right)$ such that $f_{u}^{\prime}(x, u) \geq \beta(x)+\alpha(x)$ for all $x \in \mathbb{R}^{N}$ and $u$ with $|u| \geq \tau$. Then there is a constant $\kappa=\kappa(\tau, \beta, C)$ such that for each solution $u$ of (1.1), $\|u\|_{L^{\infty}} \leq \kappa$ provided $\mu(u) \leq i(\beta)-1$.

Proof. We also make a indirect argument. Assume for any $n$, there exists $f_{n}$ as in Lemma 2.2 and $u_{n}$ satisfies (2.1) such that $\mu\left(u_{n}\right) \leq i(\beta)-1$ and $\left\|u_{n}\right\|_{L^{\infty}} \geq n$. Then similar to the proof of Lemma 2.2, $u_{n}(x) \rightarrow \infty$, almost everywhere in $\mathbb{R}^{N}$. For each $z \in E^{-}(\beta) \backslash\{0\}$ and by Fatou's Lemma, we have

$$
\begin{aligned}
\limsup _{j \rightarrow \infty}\left\langle I_{n}^{\prime \prime}\left(u_{n}\right) z, z\right\rangle \\
\quad=\int_{\mathbb{R}^{N}}\left(|\nabla z|^{2}+\alpha(x) z^{2}\right) d x+\limsup _{j \rightarrow \infty}\left(-\int_{\mathbb{R}^{N}} f_{n, u}^{\prime}\left(x, u_{n}(x)\right) z^{2}(x) d x\right) \\
\quad=\int_{\mathbb{R}^{N}}\left(|\nabla z|^{2}+\alpha(x) z^{2}\right) d x-\liminf _{j \rightarrow \infty} \int_{\mathbb{R}^{N}} f_{n, u}^{\prime}\left(x, u_{n}(x)\right) z^{2}(x) d x \\
\leq \int_{\mathbb{R}^{N}}\left(|\nabla z|^{2}+\alpha(x) z^{2}\right) d x-\int_{\mathbb{R}^{N}} \liminf _{j \rightarrow \infty} f_{n, u}^{\prime}\left(x, u_{n}(x)\right) z^{2}(x) d x \\
\leq \int_{\mathbb{R}^{N}}\left(|\nabla z|^{2}+\alpha(x) z^{2}\right) d x-\int_{\mathbb{R}^{N}}(\alpha(x)+\beta(x)) z^{2} d x \\
\quad=\int_{\mathbb{R}^{N}}\left(|\nabla z|^{2} d x-\int_{\mathbb{R}^{N}} \beta(x) z^{2} d x<0\right.
\end{aligned}
$$

Henceforth, for $n \geq n_{0}$, where $n_{0}$ large enough $\left\langle I_{n}^{\prime \prime}\left(u_{n}\right) z, z\right\rangle<0$. Thus we have $\mu\left(u_{n}\right) \geq i(\beta)$ for $n \geq n_{0}$, a contradiction.

Now we can give proof of Theorem 1.3.

Proof of Theorem 1.3. First we transform problem (1.1) into a nonresonant case at infinity. Then we study the non-resonant problem by Morse theory. Finally, combining Lemmas 2.2 and 2.3, we obtain solutions of problem (1.1). We consider the case where $\left(\mathrm{H}_{3}\right)$ holds at the first place. 
Without loss of generality, we can assume that $n\left(\beta_{\infty}\right)=0$. Let $\left\{\xi_{n}\right\}$ be an increasing sequence satisfying $\xi_{1}>\tau$ and $\xi_{n} \rightarrow \infty$ as $n \rightarrow \infty$.

To modify problem (1.1), we define a function $f_{n}(x, u)$ as

$$
f_{n}(x, u)=\int_{0}^{u} f_{n, u}^{\prime}(x, s) d s
$$

where

$$
\left.f_{n, u}^{\prime}(x, u)\right)= \begin{cases}f_{u}^{\prime}(x, u), & |u| \leq \xi_{n}, \\ \left(2-\frac{u}{\xi_{n}}\right) f_{u}^{\prime}(x, u)+\left(\frac{u}{\xi_{n}}-1\right) \beta_{\infty}(x), & \xi_{n}<u<2 \xi_{n}, \\ \left(2+\frac{u}{\xi_{n}}\right) f_{u}^{\prime}(x, u)-\left(\frac{u}{\xi_{n}}+1\right) \beta_{\infty}(x), & -2 \xi_{n}<u<-\xi_{n}, \\ \beta_{\infty}(x), & |u| \geq 2 \xi_{n} .\end{cases}
$$

Then $f_{n}(x, u) \in C\left(\mathbb{R}^{N}, \mathbb{R}\right)$. Now consider the following equation

$$
-\Delta u+\alpha(x) u=f_{n}(x, u), \quad x \in \mathbb{R}^{N},
$$

and the corresponding functional is

$$
\left.I_{n}(u)=\frac{1}{2} \int_{\mathbb{R}^{N}}\left(|\nabla u|^{2}+\alpha(x) u^{2}\right) d x-\int_{\mathbb{R}^{N}} F_{n}(x, u)\right) d x .
$$

It is easy to see $u=0$ is a trivial critical point of $I_{n}$. Observe that $n\left(\beta_{\infty}\right)=0$, $I_{n}$ satisfies (PS) condition by Lemma 2.1. Jointed by $\left(\mathrm{H}_{3}\right), I_{n}$ has nontrivial critical points. Let $a, b$ with $a<b$ be numbers such that any critical point $u$ of $I_{n}$ satisfies $a<I_{n}(u)<b$. What we want to verify is that there exists a nontrivial critical point $u_{n}$ of $I_{n}$ whose Morse index satisfies

$$
\mu\left(u_{n}\right)+\nu\left(u_{n}\right) \geq i\left(\beta_{\infty}\right)+1 .
$$

We prove this by an indirect method. First, if $I_{n}$ has only a finite number of critical points, by $\left(\mathrm{H}_{3}\right), i_{0}=i\left(f_{u}^{\prime}(x, 0)\right) \geq i\left(\beta_{\infty}\right)+2$. By [4], [15], we have

$$
B_{q}=\delta_{q, i\left(\beta_{\infty}\right)}
$$

and

$$
M_{q}= \begin{cases}1, & q=i_{0}, \\ 0, & q \in\left\{i\left(\beta_{\infty}\right)+1, i\left(\beta_{\infty}\right)+2, \ldots\right\} \backslash\left\{i_{0}\right\} .\end{cases}
$$

Morse inequality at the level $\left(i_{0}+1\right)$-th can be written as

$$
M_{i_{0}+1}-M_{i_{0}}+\ldots+(-1)^{i_{0}+1} M_{0} \geq B_{i_{0}+1}-B_{i_{0}}+\ldots+(-1)^{i_{0}+1} B_{0} .
$$

Also, by Morse inequality, we have

$$
\begin{aligned}
M_{i\left(\beta_{\infty}\right)}-M_{i\left(\beta_{\infty}\right)-1}+\ldots+ & (-1)^{i\left(\beta_{\infty}\right)} M_{0} \\
& \geq B_{i\left(\beta_{\infty}\right)}-B_{i\left(\beta_{\infty}\right)-1}+\ldots+(-1)^{i\left(\beta_{\infty}\right)} B_{0},
\end{aligned}
$$




$$
\begin{aligned}
M_{i\left(\beta_{\infty}\right)+1}-M_{i\left(\beta_{\infty}\right)}+\ldots & +(-1)^{i\left(\beta_{\infty}\right)+1} M_{0} \\
& \geq B_{i\left(\beta_{\infty}\right)+1}-B_{i\left(\beta_{\infty}\right)}+\ldots+(-1)^{i\left(\beta_{\infty}\right)+1} B_{0} .
\end{aligned}
$$

Notice that $M_{i\left(\beta_{\infty}\right)+1}=B_{i\left(\beta_{\infty}\right)+1}=0$. Thus (2.8) and (2.9) yield that

$$
\begin{aligned}
M_{i\left(\beta_{\infty}\right)}-M_{i\left(\beta_{\infty}\right)-1}+\ldots+ & (-1)^{i\left(\beta_{\infty}\right)} M_{0} \\
& =B_{i\left(\beta_{\infty}\right)}-B_{i\left(\beta_{\infty}\right)-1}+\ldots+(-1)^{i\left(\beta_{\infty}\right)} B_{0} .
\end{aligned}
$$

It follows from $(2.5),(2.6)$ and $(2.8)-(2.10)$ that $(2.7)$ can be reduced to

$$
\begin{aligned}
M_{i_{0}+1}-M_{i_{0}}+\ldots+(-1)^{i_{0}-i\left(\beta_{\infty}\right)-1} M_{i\left(\beta_{\infty}\right)+2} & \\
& \geq B_{i_{0}+1}-B_{i_{0}}+\ldots+(-1)^{i_{0}-i\left(\beta_{\infty}\right)-1} B_{i\left(\beta_{\infty}\right)+2}
\end{aligned}
$$

That is $-1 \geq 0$, a contradiction.

Next, if $I_{n}$ has infinitely many critical points. Let $\mathcal{K}=\left\{u \neq 0 \mid I_{n}^{\prime}(u)=0\right\}$. Then, by the Marino-Prodi argument in Section 3 of [20], for any $0<\varepsilon, \eta \ll 1$, there exists a functional $J$ such that

$$
\begin{aligned}
& \left\|I_{n}-J\right\|_{C^{2}\left(H_{0}^{1}\left(\mathbb{R}^{N}\right)\right)}<\varepsilon \\
& \left.I_{n}(u)=J(u), \quad u \in H_{0}^{1}\left(\mathbb{R}^{N}\right)\right) \backslash \mathcal{N}_{2 \eta}(\mathcal{K}) \\
& I_{n}^{\prime \prime}(u)=J^{\prime \prime}(u), \quad u \in \mathcal{N}_{\eta}(\mathcal{K})
\end{aligned}
$$

Moreover, $J$ satisfies (PS) condition and has only a finite number of critical points, which are all non-degenerate and contained in $\mathcal{N}_{\eta}(\mathcal{K})$, where $\mathcal{N}_{\eta}(\mathcal{K})$ is the domain of $\mathcal{K}$ with radius $\eta$. Therefore the Morse index of any nontrivial critical point of $J$ is at least $i\left(\beta_{\infty}\right)$ by $(2.13)$. Since $J$ satisfies (PS) condition, $\mathcal{K}$ is compact and we can also let $\eta$ converge to zero. Thus if we use $J, a-\varepsilon$ and $b+\varepsilon$ in $M_{q}$ and $B_{q}$ instead of $I_{n}, a$ and $b$ respectively, then (2.5) and (2.6) still hold and a contradiction takes place by $(2.11),(2.12)$ and the proof for $I_{n}$ with finite number of critical points.

By above discussion, $I_{n}$ has a nontrivial critical point $u_{n}$ satisfying (2.4). Then by Lemma 2.2 , there exists $\kappa>0$ such that $\left\|u_{n}\right\|_{L^{\infty}}<\kappa$. Note that $\left|u_{n}\right|<\left\|u_{n}\right\|_{L^{\infty}}<\kappa$ and $\xi_{n}$ defined above is an increasing sequence. Hence for some $n, \xi_{n}>\kappa$, which makes $f_{n, u}^{\prime}(x, u)=f_{u}^{\prime}(x, u)$ and $u_{n}$ is a nontrivial critical point of $I$, i.e. $u_{n}$ is a nontrivial solution for (1.1).

Most of the proof for assumption $\left(\mathrm{H}_{4}\right)$ is similar to the argument for assumption $\left(\mathrm{H}_{3}\right)$. Here we only give a skeleton of the proof. If the result is false, we can also get contradiction from Morse inequality. Therefore, there exists a nontrivial solution $u_{n}$ for (2.3) satisfying assumptions in Lemma 2.3. Finally by Lemma 2.3, $u_{n}$ must be a nontrivial solution for (1.1). 


\section{REFERENCES}

[1] T. BARTSCh AND Y. Ding, On a nonlinear Schrödinger equation with periodic potential, Math. Ann. 313 (1999), 15-37.

[2] T. Bartsch, Z. LiU And T. Weth, Sign changing solutions of superlinear Schrödinger Equations, Comm. Partial Differential Equations 29 (2004), 25-42.

[3] T. BARTSCH AND Z. WANG, Existence and multiplicity results for some superlinear elliptic problems on $\mathbb{R}^{N}$, Comm. Partial Differential Equations 20 (1995), 1725-1741.

[4] K.C. Chang, Infinite-Dimensional Morse Theory and Multiple Solution Problems, Progress in Nonlinear Differential Equations and their Applications, No. 6, Birkhäuser, Boston, 1993, 36-38.

[5] D.G. Costa, On a class of elliptic systems in $\mathbb{R}^{N}$, Electron. J. Differential Equations 7 (1994), 1-14.

[6] Y. Ding And C. LeE, Multiple solutions of Schrödinger equations with indefinite linear part and super or asymptotically linear terms, J. Differential Equations 222 (2006), 137163.

[7] Y. Ding AND S. LuAn, Multiple solutions for a class of nonlinear Schrödinger equations, J. Differential Equations 207 (2004), 423-457.

[8] M.F. Furtado, L.A. Maia And E.A.B. Silva, On a double resonant problem in $\mathbb{R}^{N}$, Differential Integral Equations 15 (2002), 1335-1344.

[9] H.P. HeInz, On the number of solutions of nonlinear Schrödinger equations and on unique continuation, J. Differential Equations 116 (1995), 149-171.

[10] W. Kryszewski and A. Szulkin, Generalized linking theorem with an application to semilinear Schrödinger equation, Adv. Differential Equations 3 (1998), 441-472.

[11] S. LI, A new Morse theory and strong resonant problems, Topol. Methods Nonlinear Anal. 21 (2003), 81-100.

[12] S. Li AND W. Zou, The computations of the critical groups with an application to elliptic resonant problems at a higher eigenvalue, J. Math. Anal. Appl. 235 (1999), 237-259.

[13] Z. Liu, J. Su AND Z. WANG, Solutions of elliptic problems with nonlinearities of linear growth, Calc. Var. Partial Differential Equations 35 (2009), 463-480.

[14] Z. LiU AND Z. WANG, Multi-bump type nodal solutions having a prescribed number of nodal domains I, Ann. Inst. H. Poincaré 22 (2005), 597-608.

[15] J. Mawhin and M. Willem, Critical Point Theory and Hamiltonian Systems, SpringerVerlag, Berlin, 1989, 182-184.

[16] L. PISAnI, Multiple solutions for elliptic equations at resonance, NoDEA Nonlinear Differential Equations Appl. 8 (2001), 389-398.

[17] P.H. Rabinowitz, On a class of nonlinear Schrödinger equations, Z. Angew. Math. Phys. 43 (1992), 270-291.

[18] M. Ramos, S. Terracini and C. Troestler, Superlinear indefinite elliptic problems and Pohozaev type identities, J. Funct. Anal. 159 (1998), 596-628.

[19] A. Salvatore, Some multiplicity results for a superlinear elliptic probles in $\mathbb{R}^{N}$, Topol. Methods Nonlinear Anal. 21 (2003), 29-39.

[20] S. Solimini, Morse index estimates in min-max theorems, Manuscripta Math. 63 (1989), 421-453.

[21] A. Szulkin and T. Weth, Ground state solutions for some indefinite variational problems, J. Funct. Anal. 257 (2009), 3802-3822.

[22] M. Willem, Minimax Theorems, Birkhäuser, Boston, 1996. 
[23] W. Zou And J. Liu, Multiple Solutions for resonant elliptic equations via local linking theory and Morse theory, J. Differential Equations 170 (2001), 68-95.

Manuscript received December 6, 2012

Rong CHeng

College of Mathematics and Statistics

Nanjing University of Information Science and Technology

Nanjing 210044, P.R. CHINA

E-mail address: mathchr@163.com

JIANHUA Hu

College of Science

University of Shanghai for Science and Technology

Shanghai 200093, P.R. CHINA

E-mail address: smilydragon2004@yahoo.com.cn

TMNA : VOLUMe $46-2015-\mathrm{N}^{\mathrm{O}} 1$ 